\title{
Atypical Initial Presentations of SLE - Our Experience From A Tertiary Centre In Eastern India
}

\author{
Rajesh Kumar $^{1 *}$ and Ratul Seal ${ }^{2}$ \\ ${ }^{1}$ Department of General Medicine, Ex Assistant Professor, AIIMS Bhubaneswar and currently Associate Professor of General Medicine, AIIMS, Deoghar, India \\ ${ }^{2}$ Department of General Medicine, Junior Resident AIIMS AIIMS Bhubaneswar, India
}

*Corresponding author: Rajesh Kumar, Department of General Medicine, Associate Professor of General Medicine and Ex Assistant Professor AIIMS Bhubaneswar, Deoghar, India.

Received Date: June 01, 2021

Published Date: July 02, 2021

\section{Introduction}

Systemic Lupus Erythematosus (SLE) is the prototypical multisystem connective tissue disorder affecting persons of all age and both sexes although it is more common in women with childbearing age [1]. The disease was first described by Italian physician Rogerius in 12th century in patients presenting with malar rash, but the systemic nature of the disease was established much later in 1872 by Moric Kaposi [2]. Currently the prevalence of SLE worldwide is reported between 17 to 48 per 100000 individuals [3]. The hallmark characteristics of the disease is production of large amount of different kinds of autoantibodies detectible in blood and other body fluids. Because of its wide variety of manifestations SLE has been previously dubbed as the "great mimicker" [1]. The Systemic Lupus Collaborating Clinics (SLICC) criteria and more recently the ACR-EULAR criteria is used to make a diagnosis of SLE compiling both clinical and immunological features [4]. In terms of presenting features of SLE, the usual symptoms which we encounter in our clinical life are polyarthralgia/arthritis (58\%), fever (33\%), alopecia (32\%), discoid rash (29\%), weight loss (28\%), buccal ulcer (22\%), fatigue (18\%), pleuritic chest pain (17\%), malar rash, headache, seizures, recurrent abortion, photosensitivity, cognitive impairment etc [3]. However, time and again SLE presents with certain unusual symptoms which baffles us making it difficult and time-consuming to diagnose. There are sporadic case reports of some such presentations in the various public domains.

In this article we have put together 5 cases showcasing our experience in dealing with unusual initial presentations of SLE in our tertiary centre.

\section{Cases}

Case 1: 62 year old male patient presented to us with history of fever, knee pain for 2 months and altered sensorium for 10 days. Clinical examination showed widespread purpura and ecchymosis in trunk and extremities. Routine investigations showed anemia, thrombocytopenia, raised urea and creatinine. Peripheral blood smear showed 14-20 schistocytes per HPF. Autoantibody panel showed positivity for ANA Hep2 with homogenous pattern along with dsDNA and anti-Sm positivity. Diagnosis was made of SLE with Thrombotic thrombocytopenic purpura (TTP). Patient responded to 5 cycles of plasmapheresis along with steroids, Mycophenolate mofetil and other supportive management.

Case 2: 20 year old male patient presented to us with history of irregular fever for 2 months and right sided chest pain with difficulty in breathing for 2 days. Clinical examination showed evidence of right sided hydropneumothorax with positive shifting dullness and succussion splash on that side. CT scan of chest showed hydropneumothorax with features of pneumonitis on right side. Autoantibody panel showed positivity for ANA Hep2 with homogenous pattern along with dsDNA positivity. Diagnosis of SLE was confirmed and management started with steroids, cyclophosphamide, intercostal tube drainage and other supportive measures.

Case 3: 15 year old female patient presented with fever, multiple joint pain, rash over face and back of neck, and bleeding from nose and gums. Clinical examination detected malar rash, 
alopecia and palatal ulcers. Routine investigations showed anemia hyperferritinemia, hypertriglyceridemia and low fibrinogen levels. Autoantibody panel was positive for ANA Hep2 with coarse speckled pattern with positive anti-Sm fraction. Complement 3 and 4 levels were subnormal and direct Coomb's test was strongly positive. Infectious etiology was ruled out. Bone marrow biopsy revealed increased histiocytes with hemophagocytosis. Diagnosis of SLE with hemophagocytic lymphohistiocytosis (HLH) was clinched. Management was started with steroids, cyclosporine and hydroxychloroquine

Case 4: 20 year old female patient presented with history of fatigue, ankle and knee joint pain and swelling along with blackish discoloration of right great toe proceeded by burning pain. On clinical examination right great toe dry gangrene was detected. Autoantibody panel was positive for ANA Hep2 with coarse speckled pattern with positive anti-Sm fraction. Complement 3 and 4 levels were low. Antiphospholipid profile done in this patient was negative. MR angiography of the lower limb ruled out any obstructive cause. Treatment was started with steroid and cyclophosphamide following which progression of gangrene halted.

Case 5: 31 year old female patient presented with history of Raynaud's phenomenon, joint pain and fatigue and was on evaluation in OPD basis. She was admitted to psychiatry ward when she developed catatonic posturing. Investigations showed ANA Hep2 positivity with coarse speckled pattern with positive antiSm fraction, SSa and Ro52. ANCA was negative. MRI Brain revealed multiple small infarcts in bilateral brain suggestive of CNS vasculitis. CSF study was normal. Thyroid profile and anti TPO was normal. Patient was started on steroids and cyclophosphamide. Henceforth, there was gradual improvement of catatonia in 2 months.

\section{Discussion}

In all our cases, SLE was suspected based on clinical features and confirmed with autoantibody assay. Response to treatment was assessed with monthly follow ups in outpatient department where most of our patients showed marked improvement in symptoms and quality of life.

Although this article showcases rare manifestations of SLE, these initial presentations are not unprecedented.

There is very sparse literature regarding TTP in SLE patients. However, several studies put the incidence of TTP in SLE patients to be as low as $0.5 \%$ [5]. It is difficult to detect both conditions simultaneously as many of the features overlap [6]. In our patient increased number of schistocytes in peripheral smear, low platelet count in presence of autoantibodies related to SLE and response to plasmapheresis clinched the diagnosis.

Lupus can present with any number of lung manifestations including pruritus, pneumonia, pulmonary hemorrhage or effusion, interstitial lung disease, pulmonary hypertension; but spontaneous pneumothorax in a young patient is not a common finding. Although aetiology is uncertain, but it has been postulated that rupture of small blebs secondary to underlying pneumonitis or infarction can cause spontaneous pneumothorax [7]. There are 11 reported cases of SLE presenting with pneumothorax in adults of which 10 patients had underlying parenchymal lesion. In our young patient with chest pain, hydropneumothorax was detected on clinical examination and radiography which resolved on chest tube drainage and steroids.

SLE as an autoimmune disorder can predispose to hemophagocytic lymphohistiocytosis (HLH). Multiple studies put the worldwide incidence to be somewhere around 0.9 to $4.6 \%$ [8]. It poses a diagnostic challenge as features of HLH can often be confused with sepsis which is very common in a SLE patient on immunosuppressants. In our patient HLH was diagnosed in consensus to HLH-2004 criteria and treatment given with high dose steroids following which patient improved.

Digital gangrene is a relatively rarepresentation of SLE which may be attributed to vasospasm, vasculitis or thromboembolism usually in long standing disease or in association with antiphospholipid antibody syndrome (APLA) or Raynaud's phenomenon. There are few case reports of gangrene in SLE patients with secondary APLA $[9,10]$ but in absence of APLA digital gangrene in SLE is very rare. In our patient APLA profile was negative and patient improved on steroids and cyclophosphamide therapy.

Neuropsychiatric SLE includes a constellation of symptoms both neurological and psychiatric secondary to central nervous system involvement [11]. Catatonia as a presenting feature of NPSLE is uncommon. Usual treatment in our patient with SLE caused remission without any relapse of symptoms.

The objective of our case series is to put forward the unusual initial presentations of SLE and emphasis the need of awareness so that early detection and timely institution of appropriate treatment of this deadly disease can be achieved.

\section{Conflict of Interest}

None

\section{References}

1. Kiriakidou M, Ching CL (2020) Systemic Lupus Erythematosus. Ann Intern Med172(11): ITC81-ITC96.

2. Ben Menachem E (2010) Review article: systemic lupus erythematosus: a review for anesthesiologists. Anesth Analg 111(3): 665-676.

3. Fortuna G, Brennan MT (2013) Systemic lupus erythematosus: epidemiology, pathophysiology, manifestations, and management. Dent Clin North Am 57(4): 631-655.

4. Tunnicliffe DJ, Singh Grewal D, Kim S, Craig JC, Tong A (2015) Diagnosis, Monitoring, and Treatment of Systemic Lupus Erythematosus: A Systematic Review of Clinical Practice Guidelines. Arthritis Care Res 67(10): 1440-1452.

5. Abu Hishmeh M, Sattar A, Zarlasht F, Ramadan M, Abdel Rahman A, et al. (2016) Systemic Lupus Erythematosus Presenting as Refractory Thrombotic Thrombocytopenic Purpura: A Diagnostic and Management 
Challenge. A Case Report and Concise Review of the Literature. Am J Case Rep 17: 782-787.

6. Fayyaz A, Igoe A, Kurien BT, Danda D, James JA, et al. (2015) Haematological manifestations of lupus. Lupus Sci Med 2(1): e000078.

7. Hannah JR, D Cruz DP (2019) Pulmonary Complications of Systemic Lupus Erythematosus. Semin Respir Crit Care Med 40(2): 227-234.

8. Cron RQ Davi S, Minoia F, Ravelli A (2015) Clinical features and correct diagnosis of macrophage activation syndrome. Expert Rev Clin Immunol 11(9): 1043-1053.
9. Stewart S, Brenton Rule A, Dalbeth N, Aiyer A, Frampton C, et al. (2019) Foot and ankle characteristics in systemic lupus erythematosus: A systematic review and meta-analysis. Semin Arthritis Rheum 48(5): 847-859.

10. Cozzani E, Gasparini G, Papini M, Burlando M, Drago F, et al. (2015) Vasculitis associated with connective tissue diseases. G Ital Dermatol Venereol 150(2): 221-232.

11. Boeke A, Pullen B, Coppes L, Medina M, Cooper JJ (2018) Catatonia Associated With Systemic Lupus Erythematosus (SLE): A Report of Two Cases and a Review of the Literature. Psychosomatics 59(6): 523-530. 